EDITORIAL

\title{
Ischaemic preconditioning and myocardial adaptation to serial intracoronary balloon inflation: cut from the same cloth?
}

\author{
M E Faircloth, S R Redwood, M S Marber
}

Heart 2004;90:358-360. doi: 10.1136/hrt.2003.025791

Elective percutaneous coronary intervention fulfils many of the criteria needed of a clinical model of ischaemic preconditioning. But is this really a reflection of the laboratory phenomenon of ischaemic preconditioning?

$\mathrm{T}$ he term "ischaemic preconditioning" was first coined in 1986 by Murry and colleagues, ${ }^{1}$ to describe a cardioprotective phenomenon that follows brief periods of myocardial ischaemia and that manifests during subsequent prolonged ischaemia. In this original description the magnitude of protection was substantial, reducing the volume of myocardial infarct by $75-80 \%$. Over the next few years the potency and reproducibility of this phenomenon was confirmed in numerous studies conducted on perfused isolated, and in situ, animal hearts. These animal studies were designed to test whether preconditioning was initiated or prevented by various pharmacological interventions before myocardial ischaemia of sufficient duration to cause infarction. Studies with such a design could obviously not be replicated in patients. The lack of definitive studies in patients fostered uncertainty as to whether ischaemic preconditioning could be "bottled" to benefit patients. ${ }^{2}$ What was urgently needed was a robust model of ischaemic preconditioning in patients where myocardial ischaemia could be controlled and measured. Such a model could then be used to determine if the mechanisms underlying "true" preconditioning against infarction in animals could be reciprocated in patients and therefore have the potential to be translated into new treatment.

\section{IS MYOCARDIAL ADAPTATION TO SERIAL BALLOON OCCLUSION DURING PCI A MODEL OF ISCHAEMIC PRECONDITIONING?}

See end of article for authors' affiliations ....................

Correspondence to: Professor Michael S Marber, Department of Cardiology, KCL, The Rayne Institute, St Thomas' Hospital, London SEl 7EH, UK;

mike.marber@kcl.ac.uk fulfils many of the criteria needed of a clinical model of ischaemic preconditioning. The timing of myocardial ischaemia can be controlled during repeated intracoronary balloon occlusions. The severity of the consequent myocardial ischaemia can be measured by the amplitude of ST segment elevation on the surface and intracoronary ECG, segmental contractile dysfunction, frequency of ventricular dysrhythmia,
Many studies have used balloon occlusion during PCI to demonstrate that at similar durations of occlusion there is an attenuation of these manifestations of myocardial ischaemia on successive, compared to first, occlusion. The question is whether this really is a reflection of the laboratory phenomenon of ischaemic preconditioning.

There are four principle determinants of the severity of myocardial ischaemia. ${ }^{3}$ The first twoduration of ischaemia and volume of myocardium perfused beyond the target lesion-can be controlled or do not vary appreciably between successive balloon inflations, and therefore cannot explain adaptation under these circumstances. The third factor is the amount of collateral myocardial blood flow during coronary occlusion. Previous studies have attempted to control for this by including only those patients without angiographically visible collaterals and others, and we have also excluded this contribution by measuring distal intracoronary balloon occlusion pressure. ${ }^{45}$ The remaining factor is the endogenous resistance of the myocardium to ischaemia. The only phenomenon, other than hypothermia and/or cardioplegia, that can reproducibly increase endogenous myocardial resistance is ischaemic preconditioning. Thus, many investigators believe that the clinical phenomenon of myocardial adaptation to serial balloon occlusion is synonymous with the laboratory phenomenon of ischaemic preconditioning against infarction. However, if this is the case it should also share the pharmacological signature of preconditioning. The receptor and channel dependence of ischaemic preconditioning in animals has been investigated intensively and involves the opening of an ATP dependent potassium channel $\left(\mathrm{K}_{\mathrm{ATP}}\right)$, in either the sarcolemma or mitochondrion, that can be blocked by glibenclamide and opened by nicorandil and pinacidil.

"Observe due measure, for right timing is in all things the most important factor" - Hesiod $(\sim 800 \mathrm{BC})$ and/or patients' anginal discomfort.
In this issue, Lindhardt and colleagues ${ }^{6}$ have tested the $\mathrm{K}_{\mathrm{ATP}^{-}}$dependence of myocardial adaptation to repeated left anterior descending coronary artery occlusions during elective PCI carried out in the presence of pinacidil, glibenclamide, or placebo. The readouts of myocardial ischaemia were ST segment shift on the 
surface ECG, angina severity score, and left ventricular (LV) dysfunction, measured by radionucleide ejection fraction. Patients with collaterals visible on their prior diagnostic coronary angiogram were excluded. Surprisingly there was no significant difference between and within the groups with regard to the amplitude of ST segment shift, decrement in LV function, or anginal severity on first versus subsequent balloon occlusions. The authors conclude, with qualifications, that the study does "not support the notion that the human myocardium has an intrinsic protective mechanism" and therefore does not exhibit ischaemic preconditioning.

It is clear the Lindhardt study ${ }^{6}$ is carefully performed but yet the findings are, on the surface, counterintuitive and therefore particularly notable and worthy of analysis. Does the study really mean intracoronary balloon occlusion can no longer be used as a surrogate of preconditioning? This seems unlikely since a number of other studies have demonstrated myocardial adaptation to serial balloon occlusion in a manner akin to ischaemic preconditioning. ${ }^{4-11}$ Furthermore closer analysis of these studies sheds some light on the possible reasons for Lindhardt's observations. Of particular relevance is the duration of balloon inflation required to initiate subsequent adaptation.

In a key study, Matsubara and colleagues ${ }^{10}$ showed that a balloon inflation time of 60 seconds did not initiate protection whereas one of 180 seconds did. Thus it seems the duration of ischaemia needed to initiate myocardial adaptation in patients lies between $60-180$ seconds. This is wholly in keeping with the 180 seconds minimum duration of ischaemia required to initiate the ischaemic preconditioning phenomenon in animals. ${ }^{12}$ Further analyses of PCI studies suggest that the threshold for innate adaptation of the human myocardium also lies close to 180 seconds. For example, although 120 seconds of intracoronary balloon occlusion frequently elicits adaptation, ${ }^{13}{ }^{14}$ the response to inflations of 90 seconds duration are variable with both positive $^{7-915}$ and negative ${ }^{16}$ results. Even 120 seconds is likely to be insufficient in some circumstances. In common with aged animals, where more prolonged ischaemia is needed to initiate preconditioning, the myocardium of older versus younger patients ( $\geqslant 65$ years $v \leqslant 55$ years) fails to adapt after 120 seconds of intracoronary balloon occlusion but does adapt after 180 seconds. ${ }^{11}$ Taken together these observations suggest that there is a threshold duration of ischaemia below which adaptation to balloon occlusion is either not precipitated or, that if it is, there is a gradated response that is below the level of detection. Furthermore this threshold lies between 90-180 seconds, and increases with the patient's age and almost certainly with other as yet unknown factors.

In the study by Lindhart and colleagues in this issue ${ }^{6}$ the duration of balloon occlusion was 90 seconds and the mean age of the patients in the groups expected to exhibit adaptation was 60 years. Thus it is likely that the duration of balloon occlusion was too brief to trigger adaptation. In contrast to the author's interpretation one could argue that the study by Lindhardt reinforces the temporal similarities between adaptation to serial balloon occlusion in patients and ischaemic preconditioning in animals, while it is the finding of adaptation after only 90 seconds of balloon occlusion by the other investigators that is counterintuitive!

\section{HOW RELEVANT ARE $K_{\text {ATP }}$ CHANNELS TO PROTECTION OF THE HUMAN HEART?}

Another surprising finding in the study by Lindhardt and colleagues $^{6}$ is that pinacidil did not lessen the severity of myocardial ischaemia during first balloon inflation compared to placebo. As mentioned previously, $\mathrm{K}_{\text {ATP }}$ channels play a definite role in ischaemic preconditioning. However, consensus is lacking over the exact relation of these channels to the multiple, parallel, and variably redundant pathways that lead to protection. ${ }^{17}$ While two or more stimuli may initiate preconditioning when acting in concert, neither stimulus may be sufficient in isolation. This, together with other observations, suggests that preconditioning may be an "all or nothing" phenomenon. However, on balance, it is likely that instead there is a steep stimulus response curve, which masquerades as a binary response. ${ }^{17}$ This behaviour does impact on the findings of Lindhardt and colleagues. ${ }^{6}$ For example, the observed tendency for ST segment elevation to be less on second inflation within placebo and pinacidil groups is more likely to relate to the presence of adaptation in a small subset of patients rather than a partial stimulus response in the whole patient group. Such interpretation of the negative findings becomes even more tenuous when examining responses to pinacidil and placebo across patient groups. This is especially so when there are few indications, such as reductions in systemic blood pressure and increases in heart rate, that the pinacidil concentrations achieved were sufficient to open myocardial $\mathrm{K}_{\mathrm{ATP}}$ channels.

As is probably apparent in the reading of this editorial, the clinical relevance of the laboratory phenomenon of ischaemic preconditioning is as yet unclear, at least to us! This situation is little changed by the article of Lindhardt and colleagues. ${ }^{6}$ However the authors are to be congratulated on completing a carefully designed and blinded clinical study based on laboratory findings in ischaemic preconditioning, and for not letting their negative findings bias their desire for publication. Without such attempts, discussion of the relation between the laboratory phenomenon of ischaemic preconditioning and the clinical benefits of pre-infarction angina, ${ }^{18}$ insulin and glucose in acute myocardial infarction, ${ }^{19}$ and nicorandil in the IONA (impact of nicorandil in angina) study ${ }^{20}$ will be forever fated to hand waving.

\section{Authors' affiliations}

M E Faircloth, S R Redwood, M S Marber, Department of Cardiology, Division of Cardiovascular Research, GKT School of Medicine, KCL, The Rayne Institute, St Thomas' Hospital, London, UK

\section{REFERENCES}

1 Murry CE, Jennings R, Reimer KA. Preconditioning with ischemia: a delay of lethal cell injury in ischemic myocardium. Circulation 1986;74:1124-36.

2 Cohen MV, Downey JM. Ischaemic preconditioning: can the protection be bottled? Lancet 1993;342:6.

3 Reimer K, Jennings R. The "wavefront phenomenon" of myocardial ischemic cell death. II. Transmural progression of necrosis within the framework of ischemic bed size (myocardium at risk) and collateral flow. Lab Invest 1979;40:633-44

4 Edwards RJ, Redwood SR, Lambiase PD, et al. Antiarrhythmic and antiischaemic effects of angina in patients with and without coronary collaterals. Heart 2002;88:604-10

5 Lambiase PD, Edwards RJ, Cusack MR, et al. Exercise-induced ischemia initiates the second window of protection in humans independent of collateral recruitment. J Am Coll Cardiol 2003:41:1174-82.

6 Lindhardt TB, Gadsbøll N, Kelbæk H, et al. Pharmacological modulation of the ATP sensitive potassium channels during repeated coronary occlusions: no effect on myocardial ischaemia or function. Heart 2004:90:425-30.

7 Deutsch E, Berger M, Kussmaul WG, et al. Adaptation to ischemia during percutaneous transluminal coronary angioplasty. Clinical, hemodynamic, and metabolic features. Circulation 1990;82:2044-51.

8 Cribier A Korsatz L, Koning L, et al. Improved myocardial response and enhanced collateral circulation with long repetitive coronary occlusion during angioplasty. A prospective study. J Am Coll Cardiol 1992;20:578-86.

9 Tomai F, Crea F, Gaspardone A, et al. Ischemic preconditioning during coronary angioplasty is prevented by glibenclamide, a selective ATP-sensitive K+ channel blocker. Circulation 1994:90:700-5.

10 Matsubara T, Minatoguchi S, Matsuo $\mathrm{H}$, et al. Three minute, but not one minute, ischemia and nicorandil have a preconditioning effect in patients with coronary artery disease. J Am Coll Cardiol 2000;35:345-51.

11 Lee T-M, Su S-F, Chou T-F, et al. Loss of preconditioning by attenuated activation of myocardial ATP-sensitive potassium channels in elderly patients undergoing coronary angioplasty. Circulation 2002;105:334-40.

12 Tsuchida A, Liu GS, Mullane K, et al. Acadesine lowers temporal threshold for the myocardial infarct size limiting effect of preconditioning. Cardiovasc Res 1993;27:116-20. 
13 Billinger $M$, Fleish $M$, Eberli FR, et al. Is the development of myocardia tolerance to repeated ischemia in humans due to preconditioning or to collateral recruitment. J Am Coll Cardiol 1999;33:1027-35.

14 Leesar MA, Stoddard M, Ahmed M, et al. Preconditioning of human myocardium with adenosine during coronary angioplasty. Circulation 1997.95:2500-7.

15 Laskey WK, Beach D. Frequency and clinical significance of ischemic preconditioning during percutaneous coronary intervention. J Am Coll Cardiol 2003;42:998-1003

16 Dupouy P, Geschwind H, Pelle G, et al. Repeated coronary artery occlusions during routine balloon angioplasty do not induce myocardial preconditioning in humans. J Am Coll Cardiol 1996;27:1374-80.
17 Yellon DM, Downey JM. Preconditioning the myocardium: from cellular physiology to clinical cardiology. Physiol Rev 2003;83:1113-51.

18 Kloner RA, Shook T, Przyklenk K, et al. Previous angina alters in-hospital outcome in TIMI 4: a clinical correlate to preconditioning? Circulation 1995;91:37-45.

19 Malmberg K. Prospective randomised study of intensive insulin treatment on long term survival after acute myocardial infarction in patients with diabetes mellitus. BMJ 1997;314:1512-5.

20 IONA Investigators. Effect of nicorandil on coronary events in patients with stable angina: the impact of nicorandil in angina (IONA) randomised trial. Lancet 2002;359:1269-75.

\section{IMAGES IN CARDIOLOGY}

\section{Coronary thrombus in unstable angina: a moving target}

A 55 year old male patient without previous cardiac history underwent coronary angiography because of angina pectoris (Canadian Cardiovascular Society class 3) and a positive exercise test. The right coronary artery and left anterior descending artery showed only mild irregularities, but the mid right circumflex (RCX) artery showed a luminal filling defect suggestive of thrombus just proximal to a severe stenosis (panel A). Left ventricular function was normal. The procedure was ended and it was decided to perform percutaneous coronary intervention of the RCX after 24 hours of pretreatment with a glycoprotein IIb/IIIa inhibitor. However, only minutes after completion of the procedure the patient complained of severe chest pain. Repeat angiography showed an occluded RCX (panel B). It was noted that a sidebranch to the left atrium was now visible whereas it was not on the previous film, suggesting embolisation of a previously occluding thrombus. Immediate angioplasty of the RCX was performed, resulting in distal embolisation and no reflow (panel C), without any improvement after intracoronary papaverine. Subsequently, a thrombectomy device (Rescue catheter, Boston Scientific) was used in an attempt to remove thrombotic material and improve coronary flow. A red, organised thrombus $(6 \times 2 \mathrm{~mm})$ was removed (lower panel). The final result of the procedure showed TIMI 3 flow and grade 3 myocardial blush (panel D). Maximum creatine kinase-MB was $29 \mu \mathrm{g} / \mathrm{l}$, and left ventricular ejection fraction was $68 \%$ after two months.
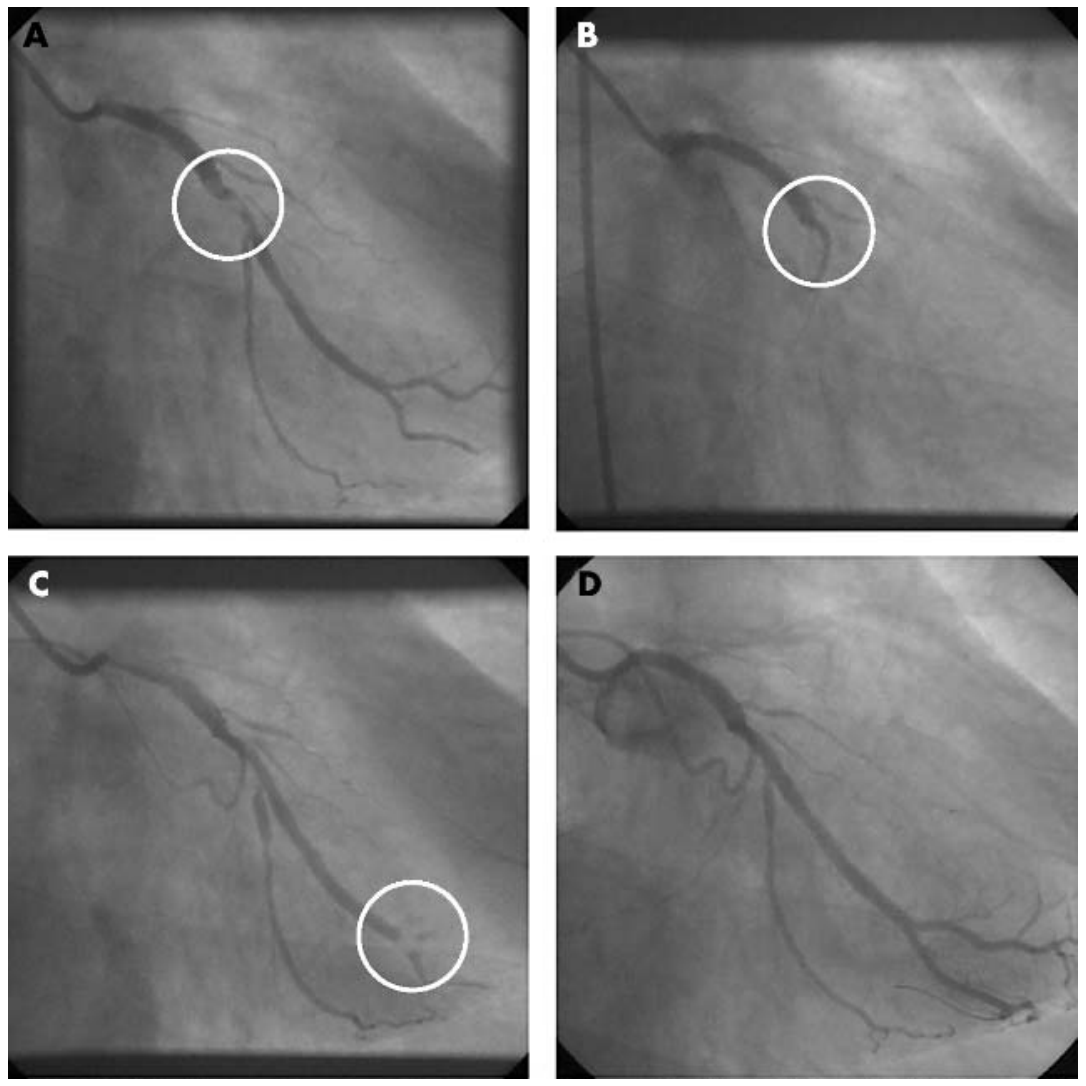

This case illustrates one of the less frequent mechanisms of acute myocardial infarction-namely, embolisation of a coronary thrombus. Thrombus formation may have occurred due to turbulent flow just proximal to a severe stenosis.

J-H E Dambrink, D M Nicastia jhe.dambrink@diagram-zwolle.nl

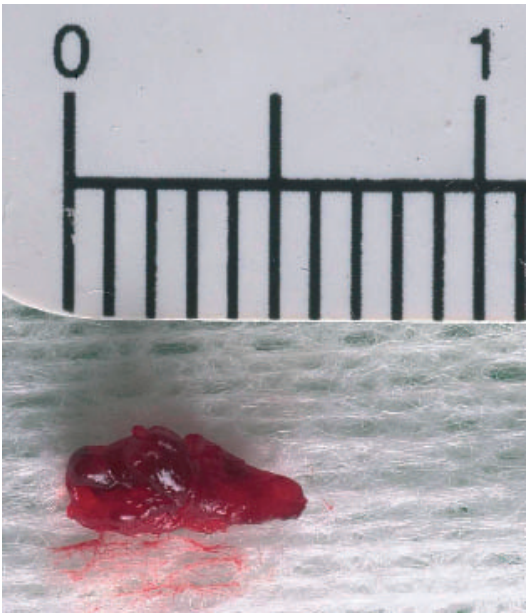

